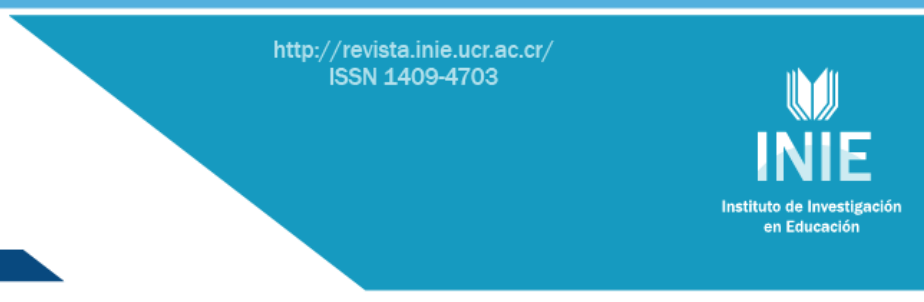

\title{
LA METACOGNICIÓN INSTITUCIONAL COMO HERRAMIENTA PARA LA MEJORA DE LA GESTIÓN DE LA CONVIVENCIA ESCOLAR
} INSTITUTIONAL METACOGNITION AS A TOOL FOR MANAGEMENT IMPROVEMENT OF SCHOOL COEXISTENCE

\section{Volumen 16, Número 3}

Setiembre-Diciembre

pp. 1-18

Este número se publicó el $1^{\circ}$ de setiembre de 2016

DOI: http://dx.doi.org/10.15517/aie.v16i3.25962

\author{
Carlos Ossa Cornejo \\ Ignacio Figueroa Céspedes \\ Felipe Rodríguez Rojas
}

Revista indizada en REDALYC, $\underline{\text { SCIELO }}$

Revista distribuida en las bases de datos:

LATINDEX, DOAJ, E-REVIST@S, IRESIE, CLASE, DIALNET, SHERPA/ROMEO, QUALIS, MIAR

Revista registrada en los directorios:

ULRICH'S, REDIE, RINACE, OEI, MAESTROTECA, PREAL, CLACSO 


\title{
LA METACOGNICIÓN INSTITUCIONAL COMO HERRAMIENTA PARA LA MEJORA DE LA GESTIÓN DE LA CONVIVENCIA ESCOLAR \\ INSTITUTIONAL METACOGNITION AS A TOOL FOR MANAGEMENT IMPROVEMENT OF SCHOOL COEXISTENCE
}

\author{
Carlos Ossa Cornejo ${ }^{1}$ \\ Ignacio Figueroa Céspedes ${ }^{2}$ \\ Felipe Rodríguez Rojas ${ }^{3}$
}

\begin{abstract}
Resumen: El presente ensayo tiene como objetivo analizar el concepto de Metacognición Institucional (MI), definido como proceso reflexivo y participativo de la comunidad educativa para enfrentar conflictos, y su uso como herramienta de gestión de la convivencia en instituciones escolares. Se realiza un análisis documental sobre las políticas de convivencia escolar predominantes en Chile, las propuestas existentes en manejo de la convivencia en el país, y acerca de la metacognición como proceso de aprendizaje organizacional. Además, se plantea que la Ml es una herramienta que incidiría en el manejo de la convivencia, la reflexión y el diálogo frente al conflicto, encauzando una toma de decisión participativa. De igual forma, se analizan las características de la MI como parte del ciclo de mejoramiento continuo de las actuales propuestas de gestión escolar. De esta forma, se concluye que, a pesar de presentar beneficios relacionados con el aprendizaje en colaboración, la diversidad y la participación, hay desafíos que obstaculizarían su aplicación para mejorar la convivencia, tanto interna, por ser una herramienta novedosa y desconocida, como externa, relacionada con las características de las instituciones escolares.
\end{abstract}

Palabras clave: CONVIVENCIA ESCOLAR, METACOGNICIÓN INSTITUCIONAL, GESTIÓN INSTITUCIONAL

Abstract: This essay aims to analyze the concept of Institutional Metacognition (IM), defined as a reflective and participating process of educative community to face conflicts and its use as a management tool of coexistence in schools. Methodological approach consists of documentary analysis of the prevailing school life policies in Chile, as well as on proposed management strategies of coexistence in schools; finally, about metacognition as institutional learning. It argues that IM is a tool that would impact on the management of coexistence, reflection, and dialogue towards the conflict, helping in participatory decision-taking. The characteristics of the IM as part of continuous improvement cycle of current proposals for diversity management are analyzed; it is done while arguing that despite having related to collaborative learning, diversity, and participation benefits, there are challenges that hinder their application to improve relationships, both internally, as a new and unknown tool, and external related features educational institutions.

Key words: SCHOOL COEXISTENCE, INSTITUTIONAL METACOGNITION, INSTITUTIONAL MANAGEMENT

\footnotetext{
1 Universidad del Bio-Bio, Chile. Psicólogo Educacional, magíster en Educación. Dirección electrónica: cossa@ubiobio.cl

2 Centro Desarrollo Cognitivo, Universidad Diego Portales, Chile. Psicólogo Educacional, magíster en Desarrollo Cognitivo. Dirección electrónica: ignacio.figueroa@mail.udp.cl

3 Dirección de Administración de Educación Municipal, Municipalidad de Pelarco, Chile. Profesor de Historia, educador diferencial, magíster en Educación. Dirección electrónica: ferodriguez85@gmail.com
}

Ensayo recibido: 6 de agosto, 2015

Enviado a corrección: 2 de mayo, 2016

Aprobado: 20 de junio, 2016 


\section{Introducción}

La política nacional de convivencia escolar del Ministerio de Educación de Chile (Mineduc) se inspira en los planteamientos de la Unesco sobre aprender a vivir juntos, formando ciudadanos y ciudadanas para una sociedad inclusiva, en paz, participativa y solidaria (Mineduc, 2011a). A través de su principal marco legal, la Ley de Violencia Escolar (Mineduc, 2011b) obliga a los establecimientos a desarrollar estrategias para promover la convivencia escolar positiva, prevenir y combatir toda forma de violencia escolar, al entregar la responsabilidad del diseño de un plan de gestión de convivencia escolar a instancias formales como los consejos escolares o comités de buena convivencia, el cual debe ser coordinado por un profesional encargado de la convivencia escolar, en colaboración y participación de todos los integrantes de la comunidad educativa. Adicionalmente, la Ley de Subvención Escolar Preferencial (Mineduc, 2009) permite la inversión de recursos financieros en la promoción y prevención de la convivencia escolar, a través de los planes de mejoramiento educativo en los establecimientos que cuentan con este beneficio.

De este modo, la política entrega un marco regulatorio acotado en términos de estructura de gestión, pero lo suficientemente amplio para que cada organización escolar pueda determinar las acciones puntuales por realizar según su realidad. Estas pueden incluir: talleres, charlas, encuentros, actividades fuera del establecimiento, cursos de capacitación, asesorías, contratación de personal especializado, etc. Sin embargo, las situaciones de violencia escolar presentes en las organizaciones escolares requieren de un enfoque que profundice en la reflexión sobre nuestro quehacer vincular y social, tanto a nivel individual como institucional. De este modo, resultarán insuficientes aquellas actuaciones de corte tradicional, como implementar normativas regulatorias y la contratación de asesorías externas sobre el tema; por el contrario, parece prioritario el generar procesos reflexivos que propicien la autorregulación colectiva y participativa.

En razón de lo anterior, este ensayo plantea una propuesta para abordar la convivencia escolar a partir del concepto de metacognición institucional (Arístegui, Bazán, Leiva, López, Muñoz y Ruz, 2005); entendida como un proceso de reflexión colaborativa entre los miembros de una organización, sobre los procesos y acciones rutinarias de la institución educativa, con el objeto de desarrollar autoconocimiento y autorregulación para el cambio en la conducta, lo cual permite confrontar, de manera reflexiva, las decisiones y acciones con la finalidad de lograr acuerdos con base en valores democráticos. 
En este ensayo, se presenta a la metacognición institucional como un dispositivo cognitivo - social que favorece un abordaje reflexivo y transformador de la convivencia en las escuelas con base en el diálogo y la participación, al destacar su relación con el ámbito de la gestión educativa.

\section{Desarrollo}

\subsection{La convivencia escolar como espacio de interacción en la escuela}

En el contexto de una escuela abierta a la diversidad, plural e inclusiva, la convivencia escolar aparece como un fenómeno de análisis, complejo y multicausal, pues se refiere precisamente al proceso de vivir con otros y otras; es decir, a la diversidad (Maldonado, 2004; Mena, Milicic, Romagnoli y Valdés 2006; Mineduc, 2004). Ha sido descrita como el conjunto de relaciones que se forman de manera cotidiana, esencial para la elaboración de vínculos de valencia afectiva, por una parte, y de lazos culturales, por otra (Maldonado, 2004). Por eso, se muestra como una construcción colectiva, culturalmente mediada y compleja, pues plantea para el sujeto la necesidad de interactuar con los demás, al tiempo de tener que conservar el espacio y la identidad personal. El Ministerio de Educación de Chile (Mineduc, 2011c) la define como:

La potencialidad que tienen las personas para vivir con otras en un marco de respeto mutuo y de solidaridad recíproca. La Convivencia Escolar se genera en la interrelación entre los diferentes miembros de la Comunidad Educativa de un establecimiento educacional que tiene incidencia significativa en el desarrollo ético, socio-afectivo e intelectual de las y los estudiantes (...) constituye una construcción colectiva y es responsabilidad de todos quienes participan del proceso educativo. (p. 14)

Para efectos de este ensayo, resulta relevante la idea de que la convivencia es producto de una construcción colectiva que se da en un marco de valores que la inspiran, lo cual resulta en interrelaciones positivas, en un contexto de respeto a la diversidad y de búsqueda del desarrollo humano. La convivencia escolar tiene repercusión en la calidad de los procesos y resultados de aprendizaje alcanzados por la organización escolar (Casassus, Cusato, Froemel y Palafox, 2001; Mena et al., 2006), al presentar múltiples impactos: influye en la calidad de los ambientes de aprendizaje en las escuelas; aumenta la calidad de los aprendizajes y mejora el rendimiento de los estudiantes; mejora en el clima escolar, con lo cual disminuye la violencia y el matonaje en el espacio escolar; incrementa el apego al 
colegio por parte del alumnado; enriquece la salud mental de quienes integran la comunidad educativa y su capacidad de resolver conflictos sin violencia; acrecenta el desarrollo socio afectivo propiamente tal; y se identifica un efecto preventivo de problemas de salud mental en docentes y estudiantes, tales como depresión, ansiedad, suicidio, desórdenes de alimentación y estrés; asimismo, impacta en la disminución de conductas de riesgo en el cuerpo estudiantil, como abuso de sustancias, violencia y actividad sexual temprana (Mena et al., 2006).

Uno de los aspectos importantes de la interacción y convivencia de las personas en la escuela es el clima social escolar. Arón y Milicic (1999) han establecido un enfoque que se trabaja de manera secundaria en las orientaciones ministeriales chilenas, centrada en el clima social escolar. Este es una dimensión dinámica de las relaciones entre los miembros de la escuela, puesto que se relaciona con la percepción poseída por docentes y educandos dentro de los procesos sociales, mientras exista acuerdo o se acepten las diferencias y reglas de funcionamiento que regulan la sociedad, el tipo de clima se estabiliza.

El clima social existente en un centro educativo siempre influirá, positiva o negativamente, en quienes integran su comunidad escolar (Mena, Romagnoli y Valdés, 2009). Por esto, cada institución debe preocupar la gestión del clima social que envuelve a sus miembros, puesto que, si una persona es asociada a un lugar donde existe uno positivo, tendrá la opción de desarrollar al máximo sus conocimientos y destrezas; además, aprenderá a enfrentar situaciones adversas, pues la existencia de un buen clima social permite un adecuado desarrollo emocional y de las habilidades sociales del individuo. Al contrario, si el que estáen el establecimiento educacional es negativo, presenciamos en estudiantes y docentes dificultades en el desarrollo de las habilidades sociales y emocionales, así como en los resultados de aprendizaje.

Se debe destacar que el clima escolar tendría relación con diversas características escolares, como el rendimiento académico, el bienestar físico y emocional de cada estudiante, y la retención escolar (Cassasus et al., 2001; Cornejo y Redondo, 2001). Se ha logrado establecer que dentro de los diversos factores contemplados por la percepción del clima escolar, uno de los factores más importantes es la relación de interacción entre las personas (Cornejo y Redondo, 2001), tanto a nivel de discentes, como en la relación entre este grupo con el cuerpo docente. Asimismo, se ve reflejado en las percepciones que tienen docentes y asistentes de la educación con la institución y las condiciones físicas esta (Vicaría para la educación, 2013). 
Milicic y Arón (2000) plantean, por una parte, la existencia de características conductuales y representacionales que configurarían distintos tipos de entornos de clima escolar, donde se generan interacciones que permiten el desarrollo de habilidades comunicativas, prosociales, de negociación, y empatía; es decir, que favorecen el desarrollo personal del estudiantado. Por otra parte, señalan también la existencia de contextos que generan interacciones en las cuales predomina el autoritarismo, la individualidad, el prejuicio y la competitividad, las que frenarían el desenvolvimiento personal de cada estudiante.

El clima escolar es un proceso que puede mejorarse a través del desarrollo de interacciones sociales positivas, así como también a través de cambios en las condiciones ambientales que promuevan satisfacción en las personas (Vicaría para la educación, 2013). Es por ello que se han planteado diferentes procedimientos para lograr mejorarlo y, a través de este, la convivencia en la escuela.

Una de las vías más relevantes propuesta por el Ministerio de Educación de Chile para promover el clima y la convivencia escolar implica el involucramiento activo del conjunto de la comunidad educativa, esto en relación con las dinámicas de producción y reproducción de las respuestas agresivas al interior de los establecimientos (Mineduc, 2011b). Un estudio dirigido por Llaña (en Mineduc, 2011a) señala que:

se observa un déficit en el logro formativo de las escuelas y se ha desperfilado el sentido y alcance de los principios orientadores; que la Convivencia Escolar debería ser entendida como un eje central que debe sostener el proyecto educativo cuya centralidad se ha perdido; que se instalan reglamentos, pero no hay, de parte de los actores, apropiación del sentido de una sana convivencia. (p. 10)

Este abordaje debiera, de acuerdo con la Ley 20.536 de Violencia Escolar chilena (Mineduc, 2011b), acompañarse de un proceso de supervisión colegiada, ya sea por el consejo escolar o por un comité de convivencia, lo que debiera implicar un proceso de análisis participativo de la comunidad educativa en la búsqueda de propuestas para favorecer la buena convivencia. En referencia a los modelos de gestión de la convivencia, Torrego (2006) distingue distintos modelos:

- El modelo sancionador, coercitivo o punitivo, que se basa en la aplicación de sanciones como principal medida para el tratamiento de la convivencia. 
- En el modelo relacional las partes en conflicto, por propia iniciativa o animados por otros, buscan la solución a sus problemas de manera que, a través del diálogo, tratan de llegar a la resolución del conflicto.

- El modelo integrado da un paso más, transcendiendo el acto privado en el que se puede convertir el acuerdo del modelo relacional puro; lo fundamental del mismo es que los procedimientos para la resolución de conflictos quedan recogidos en el reglamento de convivencia del establecimiento, generando estructuras que potencien el diálogo, como los equipos de mediación y propuestas tendientes a incrementar la participación.

El modelo integrado (Torrego, 2006) exige desarrollar en la escuela, capacidades y estructuras que potencien la reflexión y el diálogo (equipos de mediación, estructuras de participación, etc.). De este modo, se favorecen y se mejoran las relaciones interpersonales, y se potencia un ambiente de convivencia más pacífico y seguro. A su vez, implica abordar el tratamiento de conflictos para aprender a comprenderlos y enfrentarlos, al garantizar la integridad, seguridad y respeto en los miembros de la comunidad educativa.

Lo anterior implica que la comunidad escolar sea partícipe activa en la resolución de conflictos, buscando evidenciarlos para conocerlos y enfrentarlos de manera positiva.. Esa instancia se ve, sin embargo, disminuida frente a los modelos más tradicionales, caracterizados por enfoques coercitivos o relacionales dependientes de la autoridad (arbitrajes), lo que disminuye la valía de los mismos integrantes del establecimiento en el mejoramiento de sus interacciones sociales.

\subsection{Uso de Metacognición Institucional para el mejoramiento de la convivencia escolar}

Uno de los aspectos centrales en el mejoramiento de la convivencia escolar y del clima social es la necesidad de favorecer la comprensión y manejo positivo de las situaciones de conflicto presentadas en estudiantes. Permitirles conectarse con las propias vivencias y ser capaces de analizarlas de una perspectiva significativa puede ser una estrategia efectiva para originar cambios positivos, pero para ello se requieren procesos cognitivos y de regulación afectiva que operen de modo integrado y eficiente.

Diversas investigaciones realizadas en países con alto rendimiento escolar ponen de manifiesto que la efectividad de los programas e instituciones educativas tiene como gran eje 
la preocupación por los factores interaccionales que se presentan tanto entre estudiantes como entre estudiantes y docentes, así como entre estos y la familia. Esto se debe a que la escuela no es un ente aislado, sino que tiene relación con los procesos económicos, sociales, culturales y políticos que ocurren en la comunidad donde se inserta (Cassasus et al., 2001).

Desde un enfoque crítico, se ha planteado, asimismo, que un aspecto fundamental para el logro de instituciones educativas solidarias y que aceptan la diversidad tiene relación con la capacidad de modificar las pautas de interacciones, valores y procedimientos que obstaculicen la adecuada participación y atención de los integrantes de la comunidad educativa (Booth y Ainscow, 2012); por lo tanto, es tarea fundamental de la organización poder encontrar efectiva y permanentemente dichos aspectos. Lo anterior requiere de estrategias participativas y de diálogo que permitan la aparición de aspectos sensibles del clima escolar y que las situaciones de conflicto sean confrontadas para su resolución (Muñoz, Lucero, Cornejo, Muñoz y Araya, 2014).

Este proceso de abordaje de la convivencia en una institución escolar supone el uso de mecanismos de autoconocimiento y autorregulación de conductas y actitudes frente a las otras personas y frente a uno mismo. De este modo, el concepto de Metacognición Institucional (MI) se define como un proceso de reflexión colaborativa y participativa que permite a una organización completa actuar como una unidad o un conjunto para desarrollar deliberadamente procesos de autoconocimiento y autorregulación con el objetivo de mejorar sus prácticas, de forma consciente, planificada y participativa. Esto implica asumir procesos de intercambio cuyo propósito es una mejor comprensión de sus formas de interacción, sus conflictos, dificultades y potenciales (Arístegui et al., 2005).

En un sentido amplio, se debe reconocer la metacognición como una capacidad y un procedimiento que también involucra los aspectos interpersonales; esto es, a la persona y su entorno social, siendo necesario para establecer pautas interaccionales efectivas tanto para la comunidad al interior del establecimiento educacional como para su vinculación con el medio externo. Es un proceso de abordaje sistémico en términos de que su finalidad es rescatar las visiones de todos los integrantes de la comunidad educativa.

Dicha capacidad de autoconciencia de la institución debe sustentarse, además, en un diálogo participativo, elcual permita canalizar la información de los aspectos que deben ser modificados. Dicho de otro modo, la capacidad de generar conciencia sobre los procesos de la institución, y hacerlos emerger como factores de cambio, otorgaría un espacio de 
participación y valoración a los integrantes de la comunidad (Martínez, 2004; Chiu y Kuo, 2010). Esto es consistente con la mirada de la cognición distribuida, la que propone la idea de compartir información y construir conocimiento colectivamente, implicando principios tales como la colaboración y cooperación humana (Larkin, 2009; Rearte, Castillo y Sosa, 2015).

La cooperación entre personas desde esta perspectiva de cognición situada plantea que los aspectos de cognición humana son inherentes a los contextos de trabajo en los cuales participan varias personas (Salomon, 2001); esto implica, en palabras de Rogoff (1995), una apropiación participativa que se refiere a aquel proceso por el cual los individuos transforman su comprensión en el grupo a través de su propia participación en él. Desde este prisma vemos que la actividad mediada y reflexiva posee una capacidad transformadora tanto de la acción (en este caso la convivencia y el conflicto) como de la mente del sujeto participante en dicha experiencia. Esto permite ampliar la noción de aprendizaje desde la clásica transmisión de conocimientos hacia la construcción propia de ellos a partir del descubrimiento, a concebirlo como transformación de la participación (Rogoff, 1995).

Esta mirada sociocultural plantea a la metacognición como habilidad que permite reconocer y regular los procesos cognitivos entre varias personas, dado un contexto de interacción social, donde la comunicación es un puente que genera la conexión entre los pensamientos, emociones y actitudes de los y las integrantes del grupo, promoviendo el conocimiento de aquellos y aquellas y su regulación (Collazos y Mendoza, 2006). Desde esta perspectiva, la metacognición mostraría varios beneficios, pues permite organizar responsabilidades de una manera clara y equilibrada, aumenta el logro de los procesos metacognitivos individuales, y fortalece el desarrollo de estrategias de apoyo entre quienes integran el grupo o comunidad. Lo anterior se logra a través de la identificación de limitaciones en el desarrollo de tareas, coordinación de esfuerzos, aumento de comprensión de las metas y de la motivación (Chiu y Kuo, 2010; Tovar-Gálvez, 2008).

No sería un proceso solamente de evaluación o de reflexión pasiva, ya que rescata el componente de control de la acción presentado tradicionalmente por la metacognición, y permite, de esta manera, orientarla como una instancia de decisión con base en la reflexión, pero dentro de un marco colaborativo y con partición de integrantes de la comunidad escolar. De este modo, se diferencia de los procesos de evaluación y autoevaluación institucional tradicionales, pues estos se centran en medir y reflexionar a partir de pautas y criterios externamente establecidos, mientras la MI desarrolla un proceso de construcción colectiva 
de conocimientos según la intersubjetividad de los individuos para el cambio individual y colectivo de la conducta.

Así, se puede plantear la Ml como una herramienta de desarrollo institucional, relacionada con los procesos de aprendizaje organizacional, que permiten fortalecer la gestión educativa (Ossa, Castro, Castañeda y Castro, 2016). En esta misma línea, Barrero (2007) señala que la metacognición serviría para promover el mejoramiento organizacional a través de la formación basada en procesos de reflexión metacognitiva, para permitir llevar a los integrantes de la institución a un estado de mayor conciencia de sus acciones y necesidades, estado al cual no podrían llegar fácilmente por sí solos, sino con el apoyo de una persona mediadora que fomente dicho nivel de reflexión. De ese mismo modo, una institución que aprende a aprender es una que se permite tener apoyos para lograr un cambio trascendente y significativo en sus pautas y acciones frente a las necesidades dinámicas de su comunidad educativa (Stable, 2011). Para esto resulta vital el desarrollo de una racionalidad crítica y transformadora, por sobre miradas que enfaticen un mero rol técnico o práctico (Kemmis y McTaggart, 2000).

De este modo, la MI se transforma en una herramienta para evaluar, mejorar y redefinir líneas de actuación en situaciones conflictivas, y en el afrontamiento de situaciones dilemáticas; con ella se logra favorecer el trabajo cooperativo, el clima de confianza, y a través del diálogo consciente, los procesos de indagación sobre las metodologías, formas de evaluar, la vinculación entre los actores de la comunidad educativa, etc. La reflexión sobre la práctica, entonces, pretende favorecer procesos de auto-transformación a partir de cambios en la práctica cotidiana y en las concepciones y discursos personales (Kemmis y Grotenboer, 2008).

Así, el uso de la Ml como dispositivo de desarrollo de la convivencia se fundamenta en la oportunidad de contar con espacios de diálogo, reflexión y apoyo, acerca de las relaciones presentes en el establecimiento, por lo que se puede trabajar en distintos niveles y con diferentes integrantes de la comunidad educativa. Por una parte, se puede trabajar en un grupo de estudiantes, o en un curso con la ayuda de su docente, o con la persona profesional en orientación, con el fin de analizar y adecuar las interacciones entre dichos estudiantes. Por otra parte, se puede trabajar con un nivel completo, o con representantes de los cursos de un nivel, apoyados por el cuerpo docente y profesionales, analizando situaciones de interacción en dicho nivel (o entre los cursos de ese nivel), o las interacciones con otros niveles del establecimiento. Además, es posible trabajar con docentes y 
funcionarios o funcionarias para analizar situaciones conflictivas, así como de violencia, analizando pautas de interacción, visiones, políticas institucionales y procedimientos para enfrentar dichos conflictos. De igual forma, se puede incluso desarrollar actividades con madres, padres y comunidad del entorno, para ver de qué modo estos actores responden y comparten las creencias, conductas y procedimientos que la institución ha generado para la convivencia, a través de jornadas que permitan pensar la escuela para orientar su desarrollo escolar, a través de la participación y la colaboración.

En síntesis, la Ml configura una estrategia efectiva y potenciadora de la convivencia escolar al fortalecer patrones de reflexión y comunicación con el fin de identificar aquellos procesos que están en la base de las situaciones que generan conflicto entre quienes integran la comunidad escolar. Esto implica, lógicamente, una mirada de la cognición humana, culturalmente situada y construida, con el propósito de lograr una mejora en dichos elementos, con base en la concientización compartida y colaborativa.

\subsection{Utilización de la Metacognición Institucional en un ciclo de mejoramiento continuo}

Una manera de llevar a la práctica la MI, en el marco de los procesos de gestión de la organización escolar, es utilizarla en el contexto de un ciclo de mejoramiento continuo, de manera que se le otorgue una estructura lógica de aplicación, encauzada a la implementación de situaciones de mejoramiento en la organización (Mineduc, 2014). Si bien es una estrategia de gestión, no es una prerrogativa de los equipos directivos, sino una herramienta que puede ser utilizada por los distintos actores de una institución, aun cuando es mucho más útil si la emplea todo el centro educativo.

Un ciclo de mejoramiento continuo se organiza en cuatro etapas lógicas que, si bien son secuenciales, constituyen en lo fundamental una espiral ascendente de mejoramiento: determinación de la situación actual y de la situación deseada, además diseño e implementación de un plan de acción, seguimiento y evaluación (Mineduc, 2014). Esto resulta consistente con los aportes del modelo de investigación-acción participativa (Kemmis y McTaggart, 2000).

En la primera etapa, determinación de la situación actual, se busca detectar y analizar los principales problemas relacionados con la convivencia escolar en la organización, al identificar sus causas y consecuencias para la comunidad. Para esto, la Metacognición Institucional puede ser utilizada a través de un proceso de análisis y reflexión comunitaria en 
torno a los problemas de convivencia escolar presentes en la organización junto con las formas actuales de afrontamiento de estas situaciones a modo de autoconocimiento comunitario. Se pueden realizar grupos focales al interior y entre los distintos estamentos de la comunidad escolar (docentes, directivas y directivos, estudiantes, madres y padres, asistentes de la educación y sostenedor/administrador), para consensuar los problemas de convivencia de mayor impacto que afectan a la comunidad, junto con determinar sus causas, enfatizando en identificar aquellas variables susceptibles de ser intervenidas. Además, es importante que la reflexión permita diferenciar las responsabilidades colectivas y los grados de responsabilidad individual en los problemas detectados.

De la misma manera, en el desarrollo de estas experiencias, es recomendable contar con un amigo crítico (Figueroa, Soto y Sciolla, 2016). Se trata de una persona profesional de apoyo externo que facilite y profundice la reflexión en torno a los problemas de convivencia escolar, proporcionando retroalimentación con base en evidencia recopilada por aquellos equipos que abordan colegiadamente los temas ligados al aprendizaje y la participación en el establecimiento educacional (Booth y Ainscow, 2012; Figueroa y Muñoz, 2014).

Un segundo paso consiste en la determinación de la situación deseada, lo cual corresponde a tomar acuerdos como organización con respecto a qué se entenderá por una adecuada convivencia escolar, tanto en términos de metas por alcanzar como cuáles deben ser los valores y conductas esperadas en quienes integran la comunidad escolar. El uso de técnicas de educación popular o diagnóstico participativo (tales como grupos de discusión, asambleas, encuestas, ensayos y debates públicos) puede favorecer la apropiación de esta Metacognición Institucional.

La tercera etapa surge una vez que son clarificadas las situaciones actuales y deseadas, en ella la organización debe elaborar un plan de acción para mejorar la convivencia escolar. Como toda programación, debe contener acciones, responsables, plazos y recursos para su ejecución. En este aspecto, la MI puede aprovecharse mediante las mismas técnicas que en las etapas anteriores, teniendo cuidado que el plan involucre a la mayor cantidad y diversidad de individuos de la comunidad educativa.

La cuarta etapa, y quizás la más relevante para este caso, lo constituye el seguimiento y la evaluación del plan. Es aquí donde la MI, especialmente la autorregulación comunitaria, juega un rol fundamental y enriquecedor para el mejoramiento de la organización. El proceso debe realizarse en dos planos, localizado y general. En el plano localizado nos referimos al ejercicio de reflexión y autorregulación con respecto a situaciones específicas de convivencia 
escolar, referidas a la puesta en práctica del plan de acción y sus formas establecidas para enfrentar los problemas de convivencia escolar. Aquí, nuevamente, la posibilidad de contar con un amigo crítico permite colaborar con la construcción de una visión descentrada sobre aquellas acciones de promoción, prevención y abordaje de la convivencia escolar, a través de la observación y registro de situaciones o incidentes críticos relevantes de abordar.

De este modo, la Ml se ajusta de muy buena manera al proceso de gestión escolar mediante este ciclo de mejoramiento continuo, pues a través de la reflexión y diálogo constante surgen informaciones que serán analizadas y sistematizadas con el fin de evaluar el nivel de convivencia escolar, y además, de proponer acciones para mejorar los aspectos negativos que existan. Por lo tanto es una herramienta útil tanto para el diagnóstico como para el monitoreo y mejoramiento de los procesos de interacción en la institución educativa, elementos cruciales de la gestión.

\section{Síntesis y reflexiones finales}

La Ml es una herramienta innovadora para el desarrollo de procesos de cambio en la institución escolar (Arístegui et al. 2005; Ossa et al. 2016). En este ensayo se muestra la propuesta de utilizarla para el mejoramiento de la convivencia escolar. Se ha planteado que es un medio que promueve la reflexión, la participación y el diálogo en el centro escolar y, por ello, tendría un impacto en los procesos de interacción que estructuran el clima escolar y la convivencia y que se expresan en la cultura escolar.

Se señala, asimismo, que es un instrumento que aporta a la gestión educativa, pues permite pensar la escuela en relación con el abordaje de los conflictos, al proyectar propuestas para mejorar dicha respuesta. El uso de la MI desde una mirada de mejoramiento continuo permitiría elaborar propuestas de gestión de la convivencia sustentables y participativas, por lo que debiera promoverse su uso como mecanismo para enfrentar los conflictos, pues se basaría en un proceso dialógico y democrático, proceso alineado con los actuales parámetros éticos que se establecen para una gestión socialmente responsable.

Si bien creemos que la Ml es una herramienta efectiva y muy factible de llevar a cabo en establecimientos educativos para el fortalecimiento de la convivencia, no podemos dejar de señalar algunos desafíos que se requieren entrever y analizar a fin de lograr el proceso de generación de la estrategia metacognitiva como una adecuada herramienta de gestión institucional. Estos tienen relación con las dificultades emergentes al momento de desarrollar estas estrategias metacognitivas como procedimiento para tomar decisiones, dinámicas de la 
administración, y la falta de procedimientos y políticas de atención a la diversidad, elementos fundamentales para lograr una buena convivencia (Booth y Ainscow, 2012; Muñoz et al., 2014).

En primer lugar, es importante reflexionar acerca de la dificultad que conlleva el uso del pensamiento metacognitivo a nivel personal, pues es un esfuerzo que habitualmente no estamos acostumbrados a realizar. En relación con ello, se puede señalar que es también un gran impulso institucional el hecho de generar espacios de reflexión sobre las propias prácticas de interacción y, más aun, promover la reflexión y discusión de si las prácticas de la institución están permitiendo o no un adecuado abordaje de la diversidad.

Para el logro de dicha MI se debe generar un espacio de reflexión, en consonancia con un enfoque de convivencia integrativo (Torrego, 2006) y sobre la base de valores y principios inclusivos en la acción (Booth y Ainscow, 2012), de tal modo que se movilice el esfuerzo auto-evaluativo de la institución, teniendo siempre en consideración que no se trata de una revisión administrativa, sino más bien de relaciones y consistencia en las acciones desarrolladas, en la búsqueda de la apropiación participativa que menciona Rogoff (1995). Esta reflexión debe orientarse a analizar lo que se hace y no se hace frente a la convivencia, en relación con los valores declarados, al establecer instancias de diálogo y proposición para crear nuevas acciones que permitan una interacción más plena y coherente.

Asimismo, un segundo ámbito de dificultad tiene relación con las características de la institución educativa y de las políticas gubernamentales, las que en general responden a patrones y procedimientos estandarizados y jerarquizados, los cuales tienden a rigidizar dichas prácticas y procederes para facilitar la gestión. Sin embargo, esta estandarización y rigidez se vuelve un obstáculo para el proceso metacognitivo, el cual debe generar procesos de reflexión y diálogo flexibles que muevan a la toma de decisiones y al cambio de acciones, pues esta flexibilidad y la capacidad de poder reorientar las necesidades y acciones, es lo que da la base de una organización inclusiva y que responde a las necesidades humanas, como se propone en la Guía para la Inclusión Educativa o Index (Booth y Ainscow, 2012). Dicha herramienta resulta consonante con la propuesta metacognitiva, al fomentar la comprensión crítica y el desarrollo de roles de acompañamiento y apoyo que permitan reflexionar sobre los procesos internos de la institución educativa con el objetivo de transformar sus políticas, culturas y prácticas (Booth y Ainscow, 2012; Figueroa et al., 2016).

Se observa, por otra parte, que no existen habitualmente en las instituciones educativas ni a nivel de sistema general, políticas claras y compartidas para organizar las 
relaciones en el plano de los conflictos y las diferencias, que en algunas situaciones son generadas por diferencias individuales (del ser humano), pero que en otras son desarrolladas por procedimientos confusos o inexistentes y conllevan, en muchas ocasiones, a omitir requerimientos importantes para las necesidades de los integrantes de la comunidad educativa. Esta implementación de políticas es un proceso que está recién poniéndose en marcha en Chile, y apuestas como el desarrollo de equipos de mediación de conflictos, estudiantes ayudantes, círculos conversacionales, o el desarrollo de comités de convivencias aparecen como dispositivos que permiten posicionar la MI en la escuela; esto propicia una reflexión abierta y consciente acerca de la conflictividad escolar y sobre el ideal de casa de enseñanza al cual aspira la institución educativa.

Un tercer conjunto de dificultades que limitan el desarrollo de la convivencia a través de la Ml guarda relación con aspectos de gestión directiva y estilos de liderazgo. Esto sucede porque una institución donde haya un estilo directivo autoritario o que genere una cultura escolar centrada en las reglas y el control -consistente con el modelo punitivo más tradicional en las instituciones educativas de acuerdo a Torrego (2006)- no permite la reflexión, conciencia y participación que promueve la MI. Por ello, es recomendable el ejercicio de un liderazgo inclusivo, transformacional y dialógico, que promueva el cambio al interior de la institución mediante el fomento de la participación de todos los agentes en la toma de decisiones institucionales, con centro siempre en el aprendizaje del estudiantado.

Finalmente, otra gran barrera contra la que se puede enfrentar esta herramienta de gestión es el tiempo, tanto a nivel de procesos como de programación escolar, puesto que el proceso de reflexión y comunicación participativa lo consume y lleva a dejar de lado acciones administrativas y pedagógicas. Asimismo, la calendarización escolar y el acotado periodo de actividades para desarrollar el curriculum escolar puede poner en jaque el desarrollo de esta herramienta al priorizar esas actividades en contra de los procesos de reflexión compartidos, que habitualmente se evalúan como improductivos. El tiempo es un factor clave, ya que estos procesos requieren una planificación que considere ciclos continuos de mejoramiento, desde la exploración e instalación hasta la consolidación y crecimiento de las estrategias de mejora; sin embargo, debe generarse un equilibrio entre estas tareas de naturaleza técnica (tiempo/resultados) y las de naturaleza practica y crítica (fines/necesidades), como lo señalan Arístegui et al. (2005).

Es altamente necesario considerar estos desafíos para lograr que la MI sea una ayuda para el fortalecimiento de la convivencia escolar en las instituciones chilenas y 
latinoamericanas, pues las estrategias centradas solo en normativas o en intervenciones compensatorias no logran generar procesos de mejoramiento y aprendizaje en los actores de la comunidad educativa. Esto se ha considerado profundamente importante para lograr una convivencia que potencie y promueva la calidad educativa (Cassasus et al., 2001; Cornejo y Redondo, 2001) y el desarrollo escolar inclusivo (Figueroa et al., 2016).

\section{Referencias}

Arístegui, Roberto, Bazán, Domingo, Leiva, Jorge, López, Ricardo, Muñoz, Bernardo y Ruz, Juan. (2005). Hacia una Pedagogía de la Convivencia. Psykhe, 14(1), 137-150. Recuperado de http://www.scielo.cl/scielo.php?script=sci arttext\&pid=S071822282005000100011 \&lng $=$ es\&tlng=es

Arón, Ana María y Milicic, Neva. (1999). Clima social escolar y desarrollo personal. Un programa de mejoramiento. Santiago: Editorial Andrés Bello.

Barrero, Narciso. (2007). Aprendizaje metacognitivo de competencias profesionales. Educación XXI, 10, 39-60.

Booth, Tony y Ainscow, Mel. (2012). Guía para la Inclusión: Desarrollando el Aprendizaje y la Participación en las Escuelas ( $3^{\circ}$ ed.). Santiago de Chile: Ed. Creando Futuro - CSIE.

Casassus, Juan Carlos, Cusato, Sandra, Froemel, Juan Enrique y Palafox, Juan Carlos. (2001). Primer estudio internacional comparativo sobre lenguaje, matemática y factores asociados, para alumnos del tercer y cuarto grado de la educación básica. Laboratorio Latinoamericano de Evaluación de la Calidad de la Educación, Unesco. Recuperado de http://unesdoc.unesco.org/images/0014/001492/149268s.pdf

Chiu, Ming Ming, y Kuo, Sze Wing. (2010). From metacognition to social Metacognition: similarities, differences, and learning. Joumal of Education Research, 3(4), 321 - 338.

Collazos, César y Mendoza, Jair. (2006). Cómo aprovechar el aprendizaje colaborativo en el aula. Educación y Educadores, 9(2), 61-76.

Cornejo, Rodrigo y Redondo, Jesús María. (2001). El clima escolar percibido por los alumnos de enseñanza media. Una investigación en algunos liceos de la Región Metropolitana. Última Década, (15), 11-52.

Figueroa, Ignacio y Muñoz, Yolanda. (2014). La Guía para la Inclusión Educativa como herramienta de autoevaluación Institucional: Reporte de una Experiencia. Revista Latinoamericana de Inclusión Educativa, 8(2), 179-198. Recuperado de http://www.rinace.net/rlei/numeros/vol8-num2/art9.pdf

Figueroa, Ignacio, Soto, Jorge y Sciolla, Bruna. (2016). Dinámicas de recepción, apropiación y contextualización del enfoque de la Guía para la inclusión educativa en escuelas municipales de una comuna de la Región Metropolitana. Pensamiento Educativo: 
Revista de Investigación Educacional Latinoamericana, 53(1), 1-13. http://doi.org/10.7764/PEL.53.1.2016.4

Kemmis Stephen y McTaggart, Robin. (2000). Participatory Action Research: Communicative Action in the Public Sphere. En Norman Denzin y Yvonna Lincoln (Eds.), Handbook of Qualitative Research (pp. 559-603). Thousand Oaks, CA: Sage Publications.

Kemmis, Stephen y Grootenboer, Peter. (2008). Situating praxis in practice: Practice architectures and the cultural, social and material conditions for practice. In Stephen Kemmis y Tracy J. Smith (Eds.), Enabling praxis: Challenges for education (pp. 37-64). Rotterdam: Sense Publishers.

Larkin, Shirley. (2009). Socially mediated metacognition and learning to write. Thinking Skills and Creativity, 4(3), 149-159.

Maldonado, Horacio. (2004). Convivencia Escolar: Ensayos y experiencias. Buenos Aires: Lugar Editorial.

Martínez, J. Reinado. (2004). Concepción de aprendizaje, metacognición y cambio conceptual en estudiantes universitarios de psicología. (Tesis Doctoral Programa de Doctorado). Universitat de Barcelona. Recuperado de https://www.researchgate.net/profile/J Reinaldo Martinez-

Fernandez/publication/39213463 Concepcin de aprendizaje metacognicin y cambio conceptual en estudiantes universitarios de Psicologa/links/54aecd5a0cf21670b35 8730b.pdf

Mena, Isidora, Milicic, Neva, Romagnoli, Claudia y Valdés, Ana. (2006). Potenciación de la política pública de convivencia social escolar en Camino al Bicentenario. Doce Propuestas para Chile. Santiago: Ediciones Universidad Católica de Chile.

Mena, María Isidora, Romagnoli, Claudia y Valdés, Ana María. (2009). El impacto del desarrollo de habilidades socio afectivas y éticas en la escuela. Revista Electrónica Actualidades Investigativas en Educación, 9(3), 1-21. DOI http://dx.doi.org/10.15517/aie.v9i3.9507

Milicic, Neva y Arón, Ana María. (2000). Climas sociales tóxicos y climas sociales nutritivos para el desarrollo personal en el contexto escolar. Revista Psykhe, 9(2), 117-123.

Ministerio de Educación (Mineduc). (2004). Convivencia Escolar: Metodologías de trabajo para las escuelas y liceos. Comprometidos por la calidad de la educación. Material de Apoyo. Documento online. Recuperado de http://www.mineduc.cl/usuarios/convivencia escolar/doc/201104251146320.Convivenci a Escolar Metodologia de Trabajo para las Escuelas y Liceos comprometidos co n la calidad de la Educacion.pdf

Ministerio de Educación (Mineduc). (2009). Ley General de Educación N²0.370. Santiago de Chile. Recuperado de http://www.mineduc.cl/usuarios/convivencia escolar/doc/201103050142570.Ley N 20 370 Ley General de Educacion.pdf 
Ministerio de Educación (Mineduc). (2011a). Política Nacional de Convivencia Escolar, Documento Síntesis. Santiago, Chile. Recuperado de http://portales.mineduc.cl/usuarios/convivencia escolar/doc/201203262308240.Politica ssintesisConvivenciaEscolar.pdf

Ministerio de Educación (Mineduc). (2011b). Ley de Violencia Escolar № 20.536. Santiago de Chile. Recuperado de http://www.leychile.cl/Navegar?idNorma=1030087

Ministerio de Educación (Mineduc). (2011c). Conviviendo mejor en la Escuela y el Liceo. Santiago de Chile. Recuperado de http://www.mineduc.cl/usuarios/convivencia escolar/doc/201203291223210.Conviviend $\underline{\text { o.pdf }}$

Ministerio de Educación (Mineduc). (2014). Plan de Mejoramiento Educativo. Orientaciones técnicas para sostenedores y directivos escolares. Santiago de Chile. Recuperado de: http://portales.mineduc.cl/usuarios/mineduc/File/2014/PME\%20DOC\%20N1\%20VF 20 $\underline{14 . p d f}$

Muñoz, María Teresa, Lucero, Boris, Cornejo, Claudia, Muñoz, Pablo y Araya, Nelson. (2014). Convivencia y clima escolar en una comunidad educativa inclusiva de la Provincia de Talca, Chile. Revista electrónica de investigación educativa, 16(2), 16-32. Recuperado de http://www.scielo.org.mx/scielo.php?script=sci arttext\&pid=S1607$\underline{40412014000200002 \& \operatorname{lng}=\mathrm{es} \& \operatorname{lng}=\mathrm{es}}$

Ossa, Carlos, Castro, Fancy, Castañeda, María Teresa y Castro, Juana. (2016). Metacognición institucional para un aprendizaje organizacional participativo: Herramienta innovadora de gestión escolar. Rev. Gestión de la Educación, 6(1), 11-21. Recuperado de: http://revistas.ucr.ac.cr/index.php/gestedu/article/view/22718/22924

Rearte, Adriana, Castillo, Joaquín y Sosa, Mabel. (2015). Cognición Distribuida para Contextos colaborativos y de Gestión de Conocimiento Académicos usables. Trabajo realizado en el proyecto Propuesta Metodológica para el desarrollo de Interfaces de Usuario de Sistemas Colaborativos, Código C23/110. Recuperado de http://jcc2013.inf.uct.cl/wp-

content/proceedings/ECC/Cognicion\%20Distribuida\%20para\%20Contextos\%20colabor ativos\%20y\%20de\%20Gestion\%20de\%20Conocimiento\%20Academicos\%20usables.p $\underline{\mathrm{df}}$

Rogoff, Barbara. (1995). Observing sociocultural activity in three planes: Participatory appropriation, guided participation and apprenticeship. En James V. Wertsch, Pablo del Río \& Amelia Alvarez (Eds.), Sociocultural studies of mind (pp. 139-165). NY: Cambridge University Press.

Salomon, Gavriel. (2001). Cogniciones Distribuidas: consideraciones psicológicas y educativas. Madrid: Amorrortu Editores.

Stable, Yudayly. (2011). Modelo de aprendizaje organizacional para organizaciones de información. CIMED, 22(3), 237-250. 
Torrego, Juan Carlos. (2006). Modelo integrado de mejora de la convivencia. Barcelona: Editorial Graó.

Tovar-Gálvez, Julio César. (2008). Modelo metacognitivo como integrador de estrategias de enseñanza y estrategias de aprendizaje de las ciencias, y su relación con las competencias. Revista Iberoamericana de Educación, (46/7). Recuperado de http://www.rieoei.org/deloslectores/2161Tovarv2.pdf

Vicaría para la educación. (2013). Convivencia escolar: Un desafío educativo. Arzobispado de Santiago. Santiago de Chile. Recuperado de http://www.vicariaeducacion.cl/docs/libro convivencia escolar.pdf 